\title{
Symmetries and conservation laws of evolution equations via multiplier and nonlocal conservation methods
}

\author{
Emrullah Yasar and Yakup Yildirim \\ Department of Mathematics, Faculty of Arts and Sciences, Uludag University, Bursa, Turkey
}

Received: 20 May 2016, Accepted: 1 September 2016

Published online: 11 February 2017.

\begin{abstract}
In this work, we have applied a new technique which is a union of multiplier and Ibragimov's nonlocal conservation method for constructing the local conservation laws of nonlinear evolution equations. One can conclude that the higher order solutions of adjoint equation can be obtained by the multiplier functions. The Lax equation and generalized Hirota-Satsuma coupled KdV system are chosen to illustrate the effectiveness of the method. Thus, we have obtained a plenty of local (some of them are the higher order) conservation laws. The combined method presents a wider applicability for handling the conservation laws of nonlinear wave equations.
\end{abstract}

Keywords: Lax equation, generalized Hirota-Satsuma coupled KdV system, symmetry, conservation laws.

\section{Introduction}

As well known, the method of Lie symmetry is one of the most powerful method for investigating the differential equations. There exist extensive literature for this topic [1]-[3].

Lie symmetry method has a close relationship with the concept of conservation laws. Conservation laws are very important tools in the study of differential equations from mathematical as well as physical point of view.If the under study system has conservation laws then its integrability is quite possible. They are also used for existence, uniqueness and Lyapunov stability analysis and construction of numerical schemes. Moreover, conservation laws are used in obtaining the new nonlocal symmetries, nonlocal conservation laws and linerization [4].

The first study in the literature for obtaining the conservation laws using the Lie symmetries is given by E. Noether [5].In this seminal work,it is showed that for Euler-Lagrange differential equations, to each Noether symmetry associated with the Lagrangian there corresponds a conservation law which can be determined explicitly by a formula. The application of Noether's theorem depends upon the knowledge of a suitable Lagrangian ([5]-[6]). There are some new approaches in the literature about construction of conservation laws such as direct method, partial Lagrangian method, the characteristic method, the variational approach (multiplier approach) and nonlocal conservation theorem method ([7], [8]-[12]).

It is proved in [11], if a differential equation (ordinary or partial) has a symmetry then the under study equation always admits a conservation law. In order to obtain the local conservation laws, later Ibragimov [13] proposed the concept of nonlinearly self adjoint differential equations.

Very recently, Gangwei et. al [14] presented a new way for obtaining the conservation laws of partial differential equations (PDEs) with the help of combination of multiplier and nonlocal conservation method. In this new method, the 
most important point is that the solution of the adjoint equation is equivalent the multiplier function. Therefore, one can obtain local conservation laws of the system using the both method together.

In this work, we considered two famous evolution equations from the soliton theory. The first one is the Lax equation. Lax equation

$$
u_{t}+10 u u_{x x x}+20 u_{x} u_{x x}+30 u^{2} u_{x}+u_{x x x x x}=0,
$$

first appeared in the work of [15] (see also [16]) when studying nonlinear equations of evolutions with linear operators. It was found that the Lax equation belongs to the completely integrable hierarchy of higher order KdV equations. This equation have infinite sets of conservation laws [17].

On the other hand, by introducing a 4 x 4 matrix spectral problem with three potentials, Wu et al. derived a new hierarchy of nonlinear evolution equations [18].

One of typical equations in the hierarchy is the new and generalized Hirota-Satsuma coupled KdV equation

$$
\begin{array}{r}
u_{t}-\frac{u_{x x x}}{2}+3 u u_{x}-3 v_{x} w-3 v w_{x}=0, \\
v_{t}+v_{x x x}-3 u v_{x}=0, \\
w_{t}+w_{x x x}-3 u w_{x}=0 .
\end{array}
$$

Soliton solutions and Miura transformationa for this system were derived in [18]-[19], respectively.

The plan of the paper is organized as follows : In Section 2, we give briefly the description of the nonlocal conservation and multiplier methods. In addition, the relationships of both methods were emphasized. Section 3 is devoted to the conservation laws of Eq.(1) and Eq.(2) with the help of this combined method. In Section 4, some concluding remarks are given.

\section{Necessary preliminaries}

In this section, we present the notations and some of the definitions below. For the details see e.g., [4],[7], [11], [13] and [14].

Consider a sth-order PDESs of $n$ independent variables $x=\left(x^{1}, x^{2}, \ldots, x^{n}\right)$ and $m$ dependent variables $u=\left(u^{1}, u^{2}, \ldots, u^{m}\right)$

$$
E^{\alpha}\left(x, u, u_{(1)}, \ldots, u_{(s)}\right)=0, \quad \alpha=1, \ldots, m
$$

where $u_{(1)}, u_{(2)} \ldots, u_{(s)}$ denote the collections of all first, second, $\ldots$, sth-order partial derivatives, that is, $u_{i}^{\alpha}=D_{i}\left(u^{\alpha}\right), u_{i j}^{\alpha}=$ $D_{j} D_{i}\left(u^{\alpha}\right), \ldots$ respectively, with the total differentiation operator with respect to $x^{i}$ given by

$$
D_{i}=\frac{\partial}{\partial x^{i}}+u_{i}^{\alpha} \frac{\partial}{\partial u^{\alpha}}+u_{i j}^{\alpha} \frac{\partial}{\partial u_{j}^{\alpha}}+\ldots, \quad i=1, \ldots, n,
$$

where the summation convention is used whenever appropriate. As usual $\mathscr{A}$ is the vector space of differential functions of finite orders. The basic operators defined in $\mathscr{A}$ are stated below.

The $n$-tuple vector $T=\left(T^{1}, T^{2}, \ldots, T^{n}\right), T^{j} \varepsilon A, j=1, \ldots, n$ is a conserved vector of Eq. (3) if $T^{i}$ satisfies

$$
\left.D_{i} T^{i}\right|_{(3)}=0
$$


Equation (5) defines a local conservation law of system (3).Every admitted conservation laws of Eq. (3) arises from multipliers $Q^{\alpha}\left(x, u, u_{(1)}, \ldots\right)$ such that

$$
Q^{\alpha} E_{\alpha}=D_{i} T^{i}
$$

holds identically. In the multiplier approach for conservation laws, one takes the variational derivative of (6) that is,

$$
\frac{\delta}{\delta u^{\beta}}\left(Q^{\alpha} E_{\alpha}\right)=0,
$$

holds for arbitrary functions of $u\left(x^{1}, x^{2}, \ldots, x^{n}\right)$ where

$$
\frac{\delta}{\delta u^{\alpha}}=\frac{\partial}{\partial u^{\alpha}}+\sum_{s \geqslant 1}(-1)^{s} D_{i_{1}} \ldots D_{i s} \frac{\partial}{\partial u_{i_{1} \ldots i_{s}}^{\alpha}}, \quad \alpha=1, \ldots, m,
$$

is the Euler(-Lagrange) operator.

In [14], the authors modified the Ibragimov's nonlocal conservation method in terms of the multiplier function. We now present the nonlocal conservation method using the multiplier function which we described in the above. It is interesting to note that the higher order solutions of Ibragimov's adjoint equation which is consequences of the concept of nonlinearly self-adjointness can be obtained by the multiplier functions. According to [14], the solutions of the adjoint equation are constructed through the multipliers satisfying

$$
\frac{\delta}{\delta u^{\alpha}}\left(Q^{\mu} E_{\alpha}\right)=0, \quad \alpha=1, \ldots, m .
$$

For each known $Q^{\mu}$, we define a formal Lagrangian function

$$
L=Q^{\mu} E_{\alpha}
$$

and whose Euler-Lagrange equations inherit, all Lie symmetry generators (point, Lie-Backlund and nonlocal) of Eq. (3). Using the Noether theorem, we obtain the following conservation laws of Eq. (3)

$$
C^{i}=N^{i} L
$$

where $N^{i}$ is the Noether operator,

$$
N^{i}=\xi^{i}+W^{\alpha} \frac{\delta}{\delta u_{i}^{\alpha}}+\sum_{s \geqslant 1} D_{i_{1}} \ldots D_{i s}\left(W^{\alpha}\right) \frac{\partial}{\partial u_{i_{1} \ldots i_{s}}^{\alpha}}, \quad i=1, \ldots, m,
$$

and

$$
X=\xi^{i} \frac{\partial}{\partial x^{i}}+\eta^{\alpha} \frac{\partial}{\partial u^{\alpha}}
$$

is a symmetry generator of (3) and $W^{\alpha}=\eta^{\alpha}-\xi^{j} u_{j}^{\alpha}$ is the characteristic function.

In the following, for Eq.(1), $Q^{\mu}=Q$, and for system (2), we will write $\left(Q^{1}, Q^{2}, Q^{3}\right)$ as $(Q, P, K)$. For two independent variables, $\left(x^{1}, x^{2}\right)$ will be written as $(x, t)$ so that $\left(C^{1}, C^{2}\right)$ will be written as $\left(C^{x}, C^{t}\right)$.

\section{Conservation laws of Eq.}

(1) and (2). 


\subsection{Lax equation}

We first consider Lax equation (1)

$$
u_{t}+10 u u_{x x x}+20 u_{x} u_{x x}+30 u^{2} u_{x}+u_{x x x x x}=0
$$

whose Lie point symmetry generators are

$$
X_{1}=\frac{\partial}{\partial t}, X_{2}=\frac{\partial}{\partial x}, X_{3}=-\frac{5 t}{2} \frac{\partial}{\partial t}-\frac{x}{2} \frac{\partial}{\partial x}+u \frac{\partial}{\partial u} .
$$

By (7), the multipliers up to fourth-order $Q_{i}$ that lead to nontrivial conservation laws are

$$
\begin{aligned}
& Q_{1}=-30 t u^{2}-10 t u_{x x}+x, \\
& Q_{2}=-\frac{35 u^{4}}{2}-20 u^{2} u_{x x}-5 u u_{x}^{2}-2 u u_{x x x x}+u_{x} u_{x x x}-\frac{u_{x x}^{2}}{2}+\frac{u_{t x}}{2}, \\
& Q_{3}=10 u^{3}+10 u u_{x x}+5 u_{x}^{2}+u_{x x x x}, \\
& Q_{4}=3 u^{2}+u_{x x}, \\
& Q_{5}=u \\
& Q_{6}=1 .
\end{aligned}
$$

It is readily seen that each $Q_{i}(i=1, \ldots, 6)$ satisfies the adjoint equation of Eq.(1)

$$
-w_{t}-10 w_{x} u_{x x}-30 u^{2} w_{x}-10 u_{x} w_{x x}-10 u w_{x x x}-w_{x x x x x}=0 .
$$

For instance, for the multiplier $Q_{5}=u$, the left-hand side of (16) becomes

$$
-u_{t}-10 u u_{x x x}-20 u_{x} u_{x x}-30 u^{2} u_{x}-u_{x x x x x}
$$

which vanishes on Eq.(1).

On the other hand the situation is the same for higher order multipliers. For example, for the higher-order multiplier $Q_{4}$, the left-hand side of (16) becomes, after some simplification,

$$
\begin{aligned}
& -6 u\left(u_{t}+10 u u_{x x x}+20 u_{x} u_{x x}+30 u^{2} u_{x}+u_{x x x x x}\right) \\
& -\left(u_{t}+10 u u_{x x x}+20 u_{x} u_{x x}+30 u^{2} u_{x}+u_{x x x x x}\right)_{x x}
\end{aligned}
$$

which also vanishes on Eq. (1).

We now present the conserved vectors of Eq.(1) using the combined method namely multiplier function and Ibragimov's method omitting the calculations. In our scheme, we use the multipliers $Q_{1}, Q_{3}, Q_{4}, Q_{5}, Q_{6} ; Q_{2}$ is cumbersome, but the procedure would be the same.

Based on the stated multiplier and symmetry, the conserved vectors are given below.

Case 1. $Q_{6}, X_{1}$,

$$
\begin{aligned}
& C^{t}=10 u u_{x x x}+20 u_{x} u_{x x}+30 u^{2} u_{x}+u_{x x x x x}, \\
& C^{x}=-\left(30 u^{2}+10 u_{x x}\right) u_{t}-10 u_{x} u_{t x}-10 u u_{t x x}-u_{t x x x x}
\end{aligned}
$$


Case 2. $Q_{6}, X_{2}$,

$$
\begin{aligned}
& C^{t}=-u_{x}, \\
& C^{x}=u_{t}+10 u u_{x x x}+20 u_{x} u_{x x}+30 u^{2} u_{x}+u_{x x x x x}-\left(30 u^{2}+10 u_{x x}\right) u_{x}-10 u_{x} u_{x x}-10 u u_{x x x}-u_{x x x x x}
\end{aligned}
$$

Case 3. $Q_{6}, X_{3}$,

$$
\begin{aligned}
C^{t} & =-\frac{5 t}{2}\left(u_{t}+10 u u_{x x x}+20 u_{x} u_{x x}+30 u^{2} u_{x}+u_{x x x x x}\right)+u+\frac{5 t u_{t}}{2}+\frac{x u_{x}}{2} \\
C^{x} & =-\frac{x}{2}\left(u_{t}+10 u u_{x x x}+20 u_{x} u_{x x}+30 u^{2} u_{x}+u_{x x x x x}\right)+\left(u+\frac{5 t u_{t}}{2}+\frac{x u_{x}}{2}\right)\left(30 u^{2}+10 u_{x x}\right) \\
& +10 u_{x}\left(\frac{3 u_{x}}{2}+\frac{5 t u_{t x}}{2}+\frac{x u_{x x}}{2}\right)+10 u\left(2 u_{x x}+\frac{5 t u_{t x x}}{2}+\frac{x u_{x x x}}{2}\right)+3 u_{x x x x}+\frac{5 t u_{t x x x x}}{2}+\frac{x u_{x x x x x}}{2}
\end{aligned}
$$

Case 4. $Q_{5}, X_{1}$,

$$
\begin{aligned}
& C^{t}=u\left(u_{t}+10 u u_{x x x}+20 u_{x} u_{x x}+30 u^{2} u_{x}+u_{x x x x x}\right)-u u_{t} \\
& C^{x}=-u_{t}\left(u\left(20 u_{x x}+30 u^{2}\right)+u_{x x x x}\right)+u_{t x} u_{x x x}-u_{t x x}\left(10 u^{2}+u_{x x}\right)+u_{t x x x} u_{x}-u u_{t x x x x}
\end{aligned}
$$

Case 5. $Q_{5}, X_{2}$,

$$
\begin{aligned}
C^{t} & =-u u_{x} \\
C^{x} & =u\left(u_{t}+10 u u_{x x x}+20 u_{x} u_{x x}+30 u^{2} u_{x}+u_{x x x x x}\right)-u_{x}\left(u\left(20 u_{x x}+30 u^{2}\right)+u_{x x x x}\right)+u_{x x} u_{x x x}-u_{x x x}\left(10 u^{2}+u_{x x}\right) \\
& +u_{x x x x} u_{x}-u u_{x x x x}
\end{aligned}
$$

Case 6. $Q_{5}, X_{3}$

$$
\begin{aligned}
C^{t} & =-\frac{5 t u}{2}\left(u_{t}+10 u u_{x x x}+20 u_{x} u_{x x}+30 u^{2} u_{x}+u_{x x x x x}\right)+u\left(u+\frac{5 t u_{t}}{2}+\frac{x u_{x}}{2}\right) \\
C^{x} & =-\frac{x u}{2}\left(u_{t}+10 u u_{x x x}+20 u_{x} u_{x x}+30 u^{2} u_{x}+u_{x x x x x}\right)+\left(u+\frac{5 t u_{t}}{2}+\frac{x u_{x}}{2}\right)\left(u\left(20 u_{x x}+30 u^{2}\right)+u_{x x x x}\right) \\
& -\left(\frac{3 u_{x}}{2}+\frac{5 t u_{t x}}{2}+\frac{x u_{x x}}{2}\right) u_{x x x}+\left(2 u_{x x}+\frac{5 t u_{t x x}}{2}+\frac{x u_{x x x}}{2}\right)\left(10 u^{2}+u_{x x}\right)-\left(\frac{5 u_{x x x}}{2}+\frac{5 t u_{t x x x}}{2}+\frac{x u_{x x x x}}{2}\right) u_{x} \\
& +\left(3 u_{x x x x}+\frac{5 t u_{t x x x x}}{2}+\frac{x u_{x x x x x}}{2}\right) u
\end{aligned}
$$

Case 7. $Q_{4}, X_{1}$

$$
\begin{aligned}
C^{t} & =\left(3 u^{2}+u_{x x}\right)\left(u_{t}+10 u u_{x x x}+20 u_{x} u_{x x}+30 u^{2} u_{x}+u_{x x x x x}\right)-u_{t}\left(3 u^{2}+u_{x x}\right), \\
C^{x} & =-u_{t}\left(\left(3 u^{2}+u_{x x}\right)\left(30 u^{2}+20 u_{x x}\right)-u_{t x}-6 u_{x} u_{x x x}-4 u u_{x x x x}-2 u_{x x}^{2}-60 u u_{x}^{2}-30 u^{2} u_{x x}-10 u_{x x}\left(3 u^{2}+u_{x x}\right)\right. \\
& \left.+10 u\left(6 u u_{x x}+6 u_{x}^{2}+u_{x x x x}\right)\right)-u_{t x}\left(u_{t}+4 u u_{x x x}+2 u_{x} u_{x x}+30 u^{2} u_{x}+10 u_{x}\left(3 u^{2}+u_{x x}\right)-10 u\left(6 u u_{x}+u_{x x x}\right)\right) \\
& -u_{t x x}\left(10 u\left(3 u^{2}+u_{x x}\right)+6 u_{x}^{2}+6 u u_{x x}+u_{x x x x}\right)+u_{t x x x}\left(6 u u_{x}+u_{x x x}\right)-u_{t x x x x}\left(3 u^{2}+u_{x x}\right)
\end{aligned}
$$

Case 8. $Q_{4}, X_{2}$

$$
\begin{aligned}
C^{t} & =-u_{x}\left(3 u^{2}+u_{x x}\right) \\
C^{x} & =\left(3 u^{2}+u_{x x}\right)\left(u_{t}+10 u u_{x x x}+20 u_{x} u_{x x}+30 u^{2} u_{x}+u_{x x x x x}\right)-u_{x}\left(\left(3 u^{2}+u_{x x}\right)\left(30 u^{2}+20 u_{x x}\right)\right. \\
& \left.-u_{t x}-6 u_{x} u_{x x x}-4 u u_{x x x x}-2 u_{x x}^{2}-60 u u_{x}^{2}-30 u^{2} u_{x x}-10 u_{x x}\left(3 u^{2}+u_{x x}\right)+10 u\left(6 u u_{x x}+6 u_{x}^{2}+u_{x x x x}\right)\right) \\
& -u_{x x}\left(u_{t}+4 u u_{x x x}+2 u_{x} u_{x x}+30 u^{2} u_{x}+10 u_{x}\left(3 u^{2}+u_{x x}\right)-10 u\left(6 u u_{x}+u_{x x x}\right)\right) \\
& -u_{x x x}\left(10 u\left(3 u^{2}+u_{x x}\right)+6 u_{x}^{2}+6 u u_{x x}+u_{x x x x}\right)+u_{x x x x}\left(6 u u_{x}+u_{x x x}\right)-u_{x x x x x}\left(3 u^{2}+u_{x x}\right)
\end{aligned}
$$


Case 9. $Q_{4}, X_{3}$,

$$
\begin{aligned}
C^{t} & =-\frac{5 t}{2}\left(3 u^{2}+u_{x x}\right)\left(u_{t}+10 u u_{x x x}+20 u_{x} u_{x x}+30 u^{2} u_{x}+u_{x x x x x}\right)+\left(u+\frac{5 t u_{t}}{2}+\frac{x u_{x}}{2}\right)\left(3 u^{2}+u_{x x}\right) \\
C^{x} & =-\frac{x}{2}\left(3 u^{2}+u_{x x}\right)\left(u_{t}+10 u u_{x x x}+20 u_{x} u_{x x}+30 u^{2} u_{x}+u_{x x x x x}\right)+\left(u+\frac{5 t u_{t}}{2}+\frac{x u_{x}}{2}\right)\left(\left(3 u^{2}+u_{x x}\right)\left(30 u^{2}+20 u_{x x}\right)\right. \\
& \left.-u_{t x}-6 u_{x} u_{x x x}-4 u u_{x x x x}-2 u_{x x}^{2}-60 u u_{x}^{2}-30 u^{2} u_{x x}-10 u_{x x}\left(3 u^{2}+u_{x x}\right)+10 u\left(6 u u_{x x}+6 u_{x}^{2}+u_{x x x x}\right)\right)+\left(\frac{3 u_{x}}{2}+\frac{5 t u_{t x}}{2}\right. \\
& \left.+\frac{x u_{x x}}{2}\right)\left(u_{t}+4 u u_{x x x}+2 u_{x} u_{x x}+30 u^{2} u_{x}+10 u_{x}\left(3 u^{2}+u_{x x}\right)-10 u\left(6 u u_{x}+u_{x x x}\right)\right)+\left(2 u_{x x}+\frac{5 t u_{t x x}}{2}+\frac{x u_{x x x}}{2}\right)\left(10 u\left(3 u^{2}+u_{x x}\right)\right. \\
& \left.+6 u_{x}^{2}+6 u u_{x x}+u_{x x x x}\right)-\left(\frac{5 u_{x x x}}{2}+\frac{5 t u_{t x x x}}{2}+\frac{x u_{x x x x}}{2}\right)\left(6 u u_{x}+u_{x x x}\right)+\left(3 u_{x x x x}+\frac{5 t u_{t x x x x}}{2}+\frac{x u_{x x x x x}}{2}\right)\left(3 u^{2}+u_{x x}\right)
\end{aligned}
$$

Case 10. $Q_{3}, X_{1}$,

$$
\begin{aligned}
C^{t} & =\left(10 u^{3}+10 u u_{x x}+5 u_{x}^{2}+u_{x x x x}\right)\left(u_{t}+10 u u_{x x x}+20 u_{x} u_{x x}+30 u^{2} u_{x}+u_{x x x x x}\right)-u_{t}\left(10 u^{3}+10 u u_{x x}+5 u_{x}^{2}+u_{x x x x}\right), \\
C^{x} & =-u_{t}\left(\left(10 u^{3}+10 u u_{x x}+5 u_{x}^{2}+u_{x x x x}\right)\left(30 u^{2}+20 u_{x x}\right)-10 u u_{t x}-10 u_{x x}\left(10 u^{3}+10 u u_{x x}+5 u_{x}^{2}+u_{x x x x}\right)-u_{t x x x}\right) \\
& -u_{t x}\left(10 u u_{t}+10 u_{x}\left(10 u^{3}+10 u u_{x x}+5 u_{x}^{2}+u_{x x x x}\right)+u_{t x x}\right)-u_{t x x}\left(10 u\left(10 u^{3}+10 u u_{x x}+5 u_{x}^{2}+u_{x x x x}\right)-u_{t x}\right) \\
& -u_{t x x x} u_{t}-u_{t x x x x}\left(10 u^{3}+10 u u_{x x}+5 u_{x}^{2}+u_{x x x x}\right)
\end{aligned}
$$

Case 11. $Q_{3}, X_{2}$

$$
\begin{aligned}
C^{t} & =-u_{x}\left(10 u^{3}+10 u u_{x x}+5 u_{x}^{2}+u_{x x x x}\right) \\
C^{x} & =\left(10 u^{3}+10 u u_{x x}+5 u_{x}^{2}+u_{x x x x}\right)\left(u_{t}+10 u u_{x x x}+20 u_{x} u_{x x}+30 u^{2} u_{x}+u_{x x x x x}\right) \\
& -u_{x}\left(\left(10 u^{3}+10 u u_{x x}+5 u_{x}^{2}+u_{x x x x}\right)\left(30 u^{2}+20 u_{x x}\right)-10 u u_{t x}-10 u_{x x}\left(10 u^{3}+10 u u_{x x}+5 u_{x}^{2}+u_{x x x x}\right)-u_{t x x x}\right) \\
& -u_{x x}\left(10 u u_{t}+10 u_{x}\left(10 u^{3}+10 u u_{x x}+5 u_{x}^{2}+u_{x x x x}\right)+u_{t x x}\right)-u_{x x x}\left(10 u\left(10 u^{3}+10 u u_{x x}+5 u_{x}^{2}+u_{x x x x}\right)-u_{t x}\right) \\
& -u_{x x x x} u_{t}-u_{x x x x x}\left(10 u^{3}+10 u u_{x x}+5 u_{x}^{2}+u_{x x x x}\right)
\end{aligned}
$$

Case 12. $Q_{1}, X_{1}$,

$$
\begin{aligned}
C^{t} & =\left(-30 t u^{2}-10 t u_{x x}+x\right)\left(u_{t}+10 u u_{x x x}+20 u_{x} u_{x x}+30 u^{2} u_{x}+u_{x x x x x}\right)-u_{t}\left(-30 t u^{2}-10 t u_{x x}+x\right), \\
C^{x} & =-u_{t}\left(\left(-30 t u^{2}-10 t u_{x x}+x\right)\left(30 u^{2}+20 u_{x x}\right)+10 t\left(30 u^{2} u_{x x}+60 u u_{x}^{2}+10 u u_{x x x x}+30 u_{x} u_{x x x}\right.\right. \\
& \left.+20 u_{x x}^{2}+u_{t x}+u_{6 x}\right)-10 u_{x x}\left(-30 t u^{2}-10 t u_{x x}+x\right)+10 u\left(-60 t u u_{x x}-60 t u_{x}^{2}-10 t u_{x x x x}\right)-180 t u_{x x}^{2}-240 t u_{x} u_{x x} \\
& \left.-60 t u u_{x x x x}-10 t u_{6 x}\right)-u_{t x}\left(-10 t\left(u_{t}+10 u u_{x x x}+20 u_{x} u_{x x}+30 u^{2} u_{x}+u_{x x x x x}\right)+10 u_{x}\left(-30 t u^{2}-10 t u_{x x}+x\right)\right. \\
& \left.-10 u\left(-60 t u u_{x}-10 t u_{x x x}+1\right)+180 t u_{x} u_{x x}+60 t u u_{x x x}+10 t u_{5 x}\right)-u_{t x x}\left(10 u\left(-30 t u^{2}-10 t u_{x x}+x\right)\right. \\
& \left.-60 t u_{x}^{2}-60 t u u_{x x}-10 t u_{x x x x}\right)-u_{t x x x}\left(60 t u u_{x}+10 t u_{x x x}-1\right)-u_{t x x x x}\left(-30 t u^{2}-10 t u_{x x}+x\right)
\end{aligned}
$$

Case 13. $Q_{1}, X_{2}$

$$
\begin{aligned}
C^{t} & =-u_{x}\left(-30 t u^{2}-10 t u_{x x}+x\right), \\
C^{x} & =\left(-30 t u^{2}-10 t u_{x x}+x\right)\left(u_{t}+10 u u_{x x x}+20 u_{x} u_{x x}+30 u^{2} u_{x}+u_{x x x x x}\right) \\
& -u_{x}\left(\left(-30 t u^{2}-10 t u_{x x}+x\right)\left(30 u^{2}+20 u_{x x}\right)+10 t\left(30 u^{2} u_{x x}+60 u u_{x}^{2}+10 u u_{x x x x}+30 u_{x} u_{x x x}\right.\right. \\
& \left.+20 u_{x x}^{2}+u_{t x}+u_{6 x}\right)-10 u_{x x}\left(-30 t u^{2}-10 t u_{x x}+x\right)+10 u\left(-60 t u u_{x x}-60 t u_{x}^{2}-10 t u_{x x x x}\right)-180 t u_{x x}^{2}-240 t u_{x} u_{x x} \\
& \left.-60 t u u_{x x x x}-10 t u_{6 x}\right)-u_{x x}\left(-10 t\left(u_{t}+10 u u_{x x x}+20 u_{x} u_{x x}+30 u^{2} u_{x}+u_{x x x x x}\right)+10 u_{x}\left(-30 t u^{2}-10 t u_{x x}+x\right)\right. \\
& \left.-10 u\left(-60 t u u_{x}-10 t u_{x x x}+1\right)+180 t u_{x} u_{x x}+60 t u u_{x x x}+10 t u_{5 x}\right)-u_{x x x}\left(10 u\left(-30 t u^{2}-10 t u_{x x}+x\right)-60 t u_{x}^{2}\right. \\
& \left.-60 t u u_{x x}-10 t u_{x x x x}\right)-u_{x x x x}\left(60 t u u_{x}+10 t u_{x x x}-1\right)-u_{x x x x x}\left(-30 t u^{2}-10 t u_{x x}+x\right) .
\end{aligned}
$$




\subsection{Generalized Hirota-Satsuma coupled KdV equation}

We now consider generalized Hirota-Satsuma coupled KdV equation. The symmetries and multipliers up to second-order $\left(Q_{i}, P_{i}, K_{i}\right)$ are

$$
X_{1}=\frac{\partial}{\partial t}, X_{2}=\frac{\partial}{\partial x}, X_{3}=-v \frac{\partial}{\partial v}+w \frac{\partial}{\partial w}, X_{4}=3 t \frac{\partial}{\partial t}+x \frac{\partial}{\partial x}-2 u \frac{\partial}{\partial u}-4 v \frac{\partial}{\partial v}
$$

and

$$
\begin{gathered}
Q_{1}=-t u+\frac{x}{3}, P_{1}=t w, K_{1}=t v, Q_{2}=0, P_{2}=w_{x}, K_{2}=-v_{x}, Q_{3}=\frac{3 u^{2}}{2}-v w-\frac{u_{x x}}{2}, \\
P_{3}=-u w+w_{x x}, K_{3}=-u v+v_{x x}, Q_{4}=-u, P_{4}=w, K_{4}=v \\
Q_{5}=1, P_{5}=0, K_{5}=0
\end{gathered}
$$

respectively.

Based on the symmetry $X_{4}$ and the obtained multipliers, the components of conserved vectors given as follows.

Case 1. $\left(Q_{5}, P_{5}, K_{5}\right)$

$$
\begin{aligned}
& C^{t}=3 t\left(u_{t}-\frac{u_{x x x}}{2}+3 u u_{x}-3 v_{x} w-3 v w_{x}\right)-2 u-x u_{x}-3 t u_{t}, \\
& C^{x}=x\left(u_{t}-\frac{u_{x x x}}{2}+3 u u_{x}-3 v_{x} w-3 v w_{x}\right)-3 u\left(2 u+x u_{x}+3 t u_{t}\right)+\frac{1}{2}\left(4 u_{x x}+x u_{x x x}+3 t u_{t x x}\right)+3 w\left(4 v+3 t v_{t}+x v_{x}\right)
\end{aligned}
$$

Case 2. $\left(Q_{4}, P_{4}, K_{4}\right)$

$$
\begin{aligned}
C^{t} & =3 t\left(-u\left(u_{t}-\frac{u_{x x x}}{2}+3 u u_{x}-3 v_{x} w-3 v w_{x}\right)+w\left(v_{t}+v_{x x x}-3 u v_{x}\right)+v\left(w_{t}+w_{x x x}-3 u w_{x}\right)\right) \\
& +u\left(2 u+3 t u_{t}+x u_{x}\right)-w\left(4 v+3 t v_{t}+x v_{x}\right)-v\left(3 t w_{t}+x w_{x}\right) \\
C^{x} & =x\left(-u\left(u_{t}-\frac{u_{x x x}}{2}+3 u u_{x}-3 v_{x} w-3 v w_{x}\right)+w\left(v_{t}+v_{x x x}-3 u v_{x}\right)+v\left(w_{t}+w_{x x x}-3 u w_{x}\right)\right) \\
& -\left(2 u+3 t u_{t}+x u_{x}\right)\left(-3 u^{2}+\frac{u_{x x}}{2}\right)+\frac{u_{x}}{2}\left(3 u_{x}+3 t u_{t x}+x u_{x x}\right)-\frac{u}{2}\left(4 u_{x x}+3 t u_{t x x}+x u_{x x x}\right) \\
& -w_{x x}\left(4 v+3 t v_{t}+x v_{x}\right)+w_{x}\left(5 v_{x}+3 t v_{t x}+x v_{x x}\right)-w\left(6 v_{x x}+3 t v_{t x x}+x v_{x x x}\right) \\
& -v_{x x}\left(3 t w_{t}+x w_{x}\right)+v_{x}\left(3 t w_{t x}+w_{x}+x w_{x x}\right)-v\left(2 w_{x x}+3 t w_{t x x}+x w_{x x x}\right)
\end{aligned}
$$

Case 3. $\left(Q_{1}, P_{1}, K_{1}\right)$

$$
\begin{aligned}
C^{t} & =3 t\left(\left(-t u+\frac{x}{3}\right)\left(u_{t}-\frac{u_{x x x}}{2}+3 u u_{x}-3 v_{x} w-3 v w_{x}\right)+t w\left(v_{t}+v_{x x x}-3 u v_{x}\right)+t v\left(w_{t}+w_{x x x}-3 u w_{x}\right)\right) \\
& -\left(2 u+3 t u_{t}+x u_{x}\right)\left(-t u+\frac{x}{3}\right)-t w\left(4 v+3 t v_{t}+x v_{x}\right)-t v\left(3 t w_{t}+x w_{x}\right), \\
C^{x} & =x\left(\left(-t u+\frac{x}{3}\right)\left(u_{t}-\frac{u_{x x x}}{2}+3 u u_{x}-3 v_{x} w-3 v w_{x}\right)+t w\left(v_{t}+v_{x x x}-3 u v_{x}\right)+t v\left(w_{t}+w_{x x x}-3 u w_{x}\right)\right) \\
& -\left(2 u+3 t u_{t}+x u_{x}\right)\left(3 u\left(-t u+\frac{x}{3}\right)+\frac{t u_{x x}}{2}\right)-\frac{1}{2}\left(-t u_{x}+\frac{1}{3}\right)\left(3 u_{x}+3 t u_{t x}+x u_{x x}\right)+\frac{1}{2}\left(-t u+\frac{x}{3}\right)\left(4 u_{x x}+3 t u_{t x x}+x u_{x x x}\right) \\
& -\left(4 v+3 t v_{t}+x v_{x}\right)\left(-3 w\left(-t u+\frac{x}{3}\right)-3 t u w+t w_{x x}\right)+t w_{x}\left(5 v_{x}+3 t v_{t x}+x v_{x x}\right)-t w\left(6 v_{x x}+3 t v_{t x x}+x v_{x x x}\right) \\
& -\left(3 t w_{t}+x w_{x}\right)\left(-3 v\left(-t u+\frac{x}{3}\right)-3 t u v+t v_{x x}\right)+t v_{x}\left(3 t w_{t x}+w_{x}+x w_{x x}\right)-t v\left(2 w_{x x}+3 t w_{t x x}+x w_{x x x}\right)
\end{aligned}
$$

Case 4. $\left(Q_{2}, P_{2}, K_{2}\right)$

$$
\begin{aligned}
C^{t} & =3 t\left(w_{x}\left(v_{t}+v_{x x x}-3 u v_{x}\right)-v_{x}\left(w_{t}+w_{x x x}-3 u w_{x}\right)\right)-w_{x}\left(4 v+3 t v_{t}+x v_{x}\right)+v_{x}\left(3 t w_{t}+x w_{x}\right), \\
C^{x} & =x\left(w_{x}\left(v_{t}+v_{x x x}-3 u v_{x}\right)-v_{x}\left(w_{t}+w_{x x x}-3 u w_{x}\right)\right)+w_{t}\left(4 v+3 t v_{t}+x v_{x}\right)+w_{x x}\left(5 v_{x}+3 t v_{t x}+x v_{x x}\right) \\
& -w_{x}\left(6 v_{x x}+3 t v_{t x x}+x v_{x x x}\right)-v_{t}\left(3 t w_{t}+x w_{x}\right)-v_{x x}\left(3 t w_{t x}+w_{x}+x w_{x x}\right)+v_{x}\left(2 w_{x x}+3 t w_{t x x}+x w_{x x x}\right)
\end{aligned}
$$


Case 5. $\left(Q_{3}, P_{3}, K_{3}\right)$,

$$
\begin{aligned}
C^{t} & =3 t\left(\left(\frac{3 u^{2}}{2}-v w-\frac{u_{x x}}{2}\right)\left(u_{t}-\frac{u_{x x x}}{2}+3 u u_{x}-3 v_{x} w-3 v w_{x}\right)+\left(-u w+w_{x x}\right)\left(v_{t}+v_{x x x}-3 u v_{x}\right)\right. \\
& \left.+\left(-u v+v_{x x}\right)\left(w_{t}+w_{x x x}-3 u w_{x}\right)\right)-\left(2 u+3 t u_{t}+x u_{x}\right)\left(\frac{3 u^{2}}{2}-v w-\frac{u_{x x}}{2}\right)-\left(4 v+3 t v_{t}+x v_{x}\right)\left(-u w+w_{x x}\right) \\
& -\left(3 t w_{t}+x w_{x}\right)\left(-u v+v_{x x}\right) \\
C^{x} & =x\left(\left(\frac{3 u^{2}}{2}-v w-\frac{u_{x x}}{2}\right)\left(u_{t}-\frac{u_{x x x}}{2}+3 u u_{x}-3 v_{x} w-3 v w_{x}\right)+\left(-u w+w_{x x}\right)\left(v_{t}+v_{x x x}-3 u v_{x}\right)\right. \\
& \left.+\left(-u v+v_{x x}\right)\left(w_{t}+w_{x x x}-3 u w_{x}\right)\right)-\left(2 u+3 t u_{t}+x u_{x}\right)\left(3 u\left(\frac{3 u^{2}}{2}-v w-\frac{u_{x x}}{2}\right)+\frac{u_{t x}}{2}-w v_{x x}-2 v_{x} w_{x}-v w_{x x}\right) \\
& -\left(3 u_{x}+3 t u_{t x}+x u_{x x}\right)\left(-\frac{u_{t}}{2}+w v_{x}+v w_{x}\right)+\frac{1}{2}\left(4 u_{x x}+3 t u_{t x x}+x u_{x x x}\right)\left(\frac{3 u^{2}}{2}-v w-\frac{u_{x x}}{2}\right) \\
& -\left(4 v+3 t v_{t}+x v_{x}\right)\left(-3 w\left(\frac{3 u^{2}}{2}-v w-\frac{u_{x x}}{2}\right)-3 u\left(-u w+w_{x x}\right)-w_{t x}+u_{x} w_{x}+2 u w_{x x}-w u_{x x}\right) \\
& -\left(5 v_{x}+3 t v_{t x}+x v_{x x}\right)\left(-2 u w_{x}+w u_{x}+w_{t}\right)-\left(6 v_{x x}+3 t v_{t x x}+x v_{x x x}\right)\left(-u w+w_{x x}\right)-\left(3 t w_{t}+x w_{x}\right)\left(-3 v\left(\frac{3 u^{2}}{2}-v w-\frac{u_{x x}}{2}\right)\right. \\
& \left.-3 u\left(-u v+v_{x x}\right)-v_{t x}+u_{x} v_{x}+2 u v_{x x}-v u_{x x}\right) \\
& -\left(3 t w_{t x}+w_{x}+x w_{x x}\right)\left(-2 u v_{x}+v u_{x}+v_{t}\right)-\left(2 w_{x x}+3 t w_{t x x}+x w_{x x x}\right)\left(-u v+v_{x x}\right) .
\end{aligned}
$$

It is readily seen that the situations are the same for the other symmetry generators.

\section{Concluding remarks}

In this work we have constructed conservation laws of the Lax equation and generalized Hirota-Satsuma coupled KdV system which is not derivable from a variational principle. Namely, no recourse to a Lagrangian formulation is made.

Using the combined approach which is a union of multiplier and Ibragimov's nonlocal conservation method, a plenty of local conservation laws were obtained.

We conclude that the solutions of adjoint equation can be obtained by the multiplier functions. The conserved vectors obtained here can be used in double reductions and solutions of the underlying equations [20].

\section{Competing interests}

The authors declare that they have no competing interests.

\section{Authors' contributions}

All authors have contributed to all parts of the article. All authors read and approved the final manuscript.

\section{References}

[1] G. W. Bluman and S. C. Anco, Symmetry and Integration Methods for Differential Equations, vol. 154 of Applied Mathematical Sciences, Springer, New York, NY, USA, 2002.

[2] P. J. Olver, Application of Lie groups to Differential Equations, Springer-Verlag, New York, 1993. 
[3] N. H. Ibragimov, CRC Handbook of Lie Group Analysis of Differential Equations, Volume 1, Symmetries, Exact Solutions and Conservation Laws., CRC Press, Boca Raton, Florida, 1995.

[4] A. F. Cheviakov, Gem software package for computation of symmetries and conservation laws of differential equations. Comput Phys Commun 2007; 176:48-61.

[5] E. Noether, Invariante Variationsprobleme, Nacr. Konig. Gesell. Wissen., Gottingen, Math.-Phys. Kl. Heft 2 (1918) 235-257 (English translation in Transport Theory and Statistical Physiscs 1 (3) (1971) 186-207)

[6] R. Naz,F. M. Mahomed, D. P. Mason, Comparison of different approaches to conservation laws for some partial differential equations in fluid mechanics, Applied Mathematics and Computation, 205 (2008) 212-230.

[7] H. Steudel, Uber die zuordnung zwischen invarianzeigenschaften und erhaltungssatzen, Z. Naturforsch. 17A (1962) 129-132.

[8] A. H. Kara, F. M. Mahomed, Relationship between symmetries and conservation laws, Int. J. Theor. Phys. 39 (2000) $23-40$.

[9] A. H. Kara, F. M. Mahomed, Noether-type symmetries and conservation laws via partial Lagragians, Nonlinear Dynam., 45 (2006) 367-383.

[10] S. C. Anco, G W Bluman, Direct construction method for conservation laws of partial differential equations. Part I: examples of conservation law classifications, Eur. J. Appl. Math., 13 (2002) 545-566.

[11] N.H. Ibragimov, A new conservation theorem, J. Math. Anal. Appl. 333 (2007) 311-328.

[12] T. Wolf, A comparison of four approaches to the calculation of conservation laws, Eur. J. Appl. Math. 13 (2002) 129-152.

[13] N. H. Ibragimov, Nonlinear self-adjointness and conservation laws, Journal of Physics A: Mathematical and Theoretical 44.43 (2011) 432002.

[14] W. Gangwei ,A. H. Kara, Conservation laws, multipliers, adjoint equations and Lagrangians for Jaulent-Miodek and some families of systems of KdV type equations, Nonlinear Dyn 81 (2015) 753-763.

[15] P. D. Lax, Integrals of nonlinear equations of evolution and solitary waves, Selected Papers Volume I (2005): 366-389.

[16] A. M. Wazwaz, The extended tanh method for new solitons solutions for many forms of the fifth-order KdV equations, Applied Mathematics and Computation 184.2 (2007): 1002-1014.

[17] U. Goktas, W. Hereman, Symbolic computation of conserved densities for systems of nonlinear evolution equations, J. Symbol. Comput. 24 (1997) 591-621.

[18] Y.T. Wu, X.G. Geng, X.B. Hu, S.M. Zhu, A generalized Hirota-Satsuma coupled Korteweg-de Vries equation and Miura transformations Phys. Lett. A 255 (1999) 259.

[19] E. Fan, Soliton solutions for a generalized Hirota-Satsuma coupled KdV equation and a coupled MKdV equation, Physics Letters A 282 (2001) 18-22

[20] A. H. Bokhari,A. Y. Al-Dweik, A. H. Kara,F. M. Mahomed,F. D. Zaman, Double reduction of a nonlinear (2+1) wave equation via conservation laws, Commun. Nonlinear Sci. Numer. Simulat., 16 (2011) 1244-1253. 